\title{
Erratum to: Prolonged thrombocytopenia after living donor liver transplantation is a strong prognostic predictor irrespective of history of splenectomy: the significance of ADAMTS13 and graft function
}

\author{
Yu Nobuoka ${ }^{1} \cdot$ Hideo Wada $^{2}$ - Shugo Mizuno ${ }^{1}$ Masashi Kishiwada ${ }^{1}$. \\ Masanobu Usui ${ }^{1} \cdot$ Hiroyuki Sakurai $^{1} \cdot$ Masami Tabata $^{1} \cdot$ Toshihiko Kobayashi $^{3}$. \\ Tsutomu Nobori ${ }^{2} \cdot$ Shinji Uemoto $^{4} \cdot$ Shuji Isaji $^{1}$
}

Published online: 21 April 2016

(c) The Japanese Society of Hematology 2016

\section{Erratum to: Int J Hematol (2014) 99:418-428 DOI 10.1007/s12185-014-1543-9}

The author would like to correct the errors in the publication of the original article. The corrected details are given below for your reading.

The original article was published with the incorrect title. The correct title should read: "Prolonged thrombocytopenia after living donor liver transplantation is a strong prognostic predictor irrespective of splenectomy: the significance of ADAMTS13 and graft function".

Secondly, the last two sentences beneath the heading "Abstract" should read as "Irrespective of splenectomy, platelet counts and ADAMTS13 activity in the LP group remained low until POD28, while VWF/ADAMTS13 ratio significantly increased until POD28. These results suggest that prolonged thrombocytopenia after LDLT was associated with not only a decrease in ADAMTS13 due to sinusoidal endothelial cell injury, but also low TPO

The online version of the original article can be found under doi:10.1007/s12185-014-1543-9.

Hideo Wada

wadahide@clin.medic.mie-u.ac.jp

1 Department of Hepatobiliary-Pancreatic and Transplant Surgery, Mie University Graduate School of Medicine, Tsu, Mie, Japan

2 Department of Molecular and Laboratory Medicine, Mie University Graduate School of Medicine, 2-174 Edobashi, Tsu, Mie 514-0001, Japan

3 Department of Hematology, Mie University Graduate School of Medicine, Tsu, Mie, Japan

4 Hepatobiliary Pancreatic and Transplantation Surgery, Kyoto University Graduate School, Kyoto, Japan

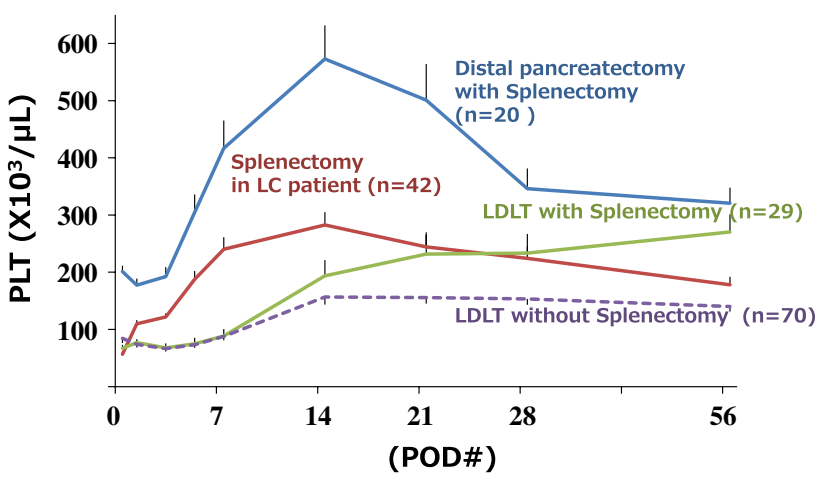

Fig. 1 Comparison of postoperative platelet counts between LDLT and other operative procedures with or without splenectomy (with standard error bars)

production due to hepatocyte dysfunction, irrespective of splenectomy."

Errors have subsequently been identified in the original publication, and the following corrections should be noted:

1. The revised Figs. 1 and 5 and Tables 1, 2, 3 and 4 were replaced with this erratum.

2. References [18] and [27] should be replaced as below.

18. Takei Y, Marzi I, Gao WS, Gores GJ, Lemasters JJ, Thurman RG. Leukocyte adhesion and cell death following orthotopic liver transplantation in the rat. Transplantation. 1991;51(5):959-65.

27. Cywes R, Mullen JB, Stratis MA, Greig PD, Levy GA, Harvey PR, Strasberg SM. Prediction of the outcome of transplantation in man by platelet adherence in donor liver allografts. Evidence of the importance of prepreservation injury. Transplantation. 1993;56(2):316-23. 

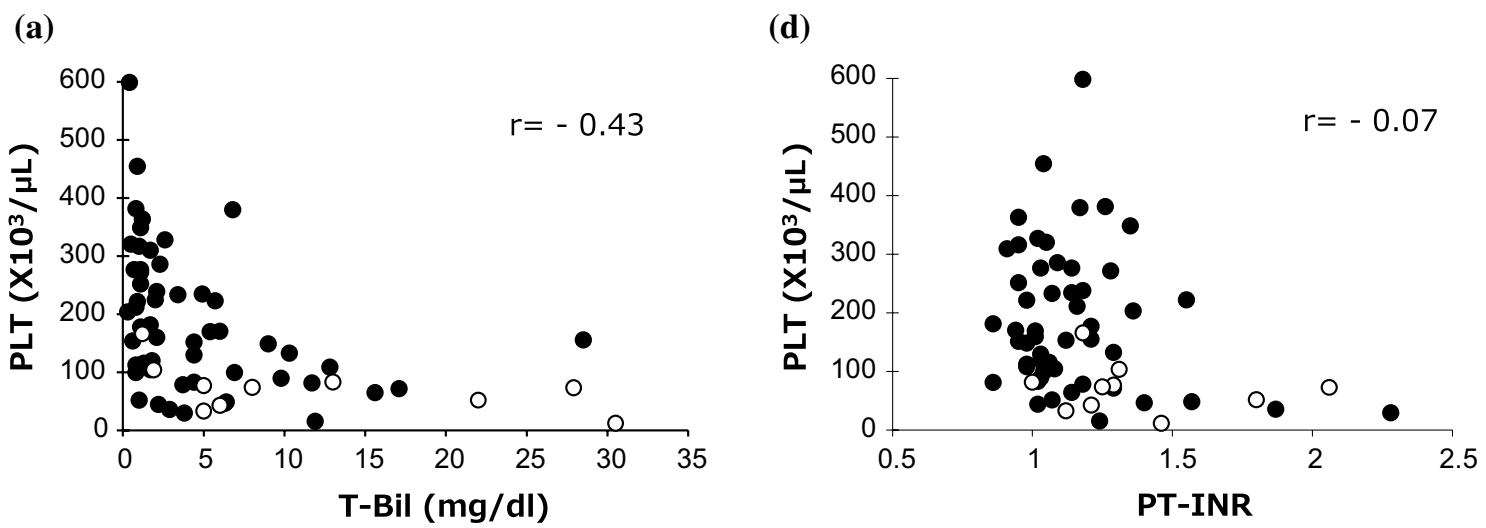

(b)

(e)
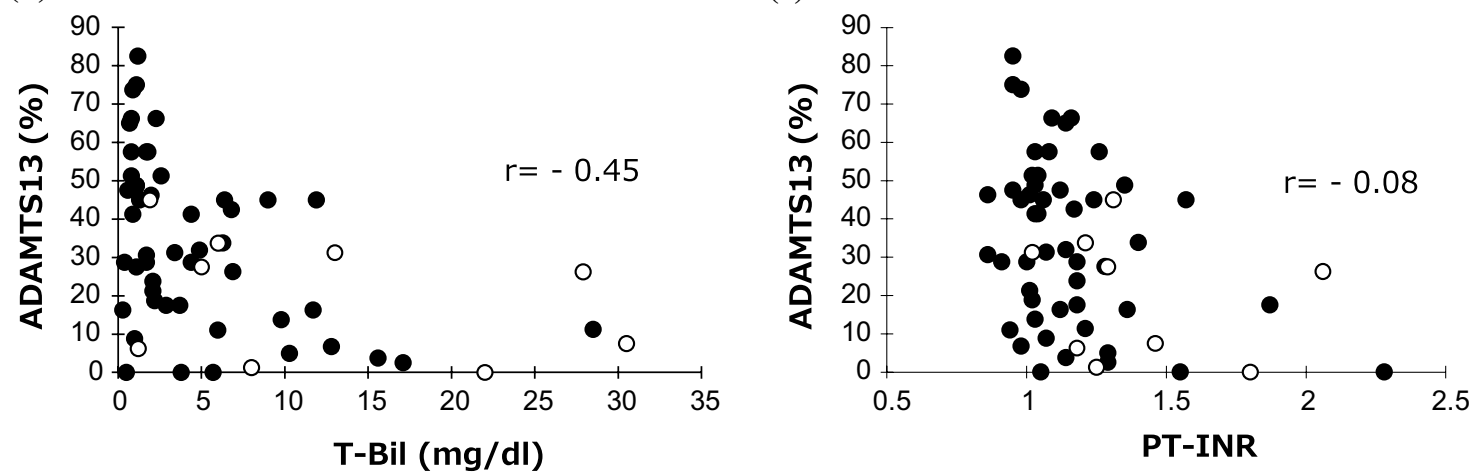

(c)

(f)
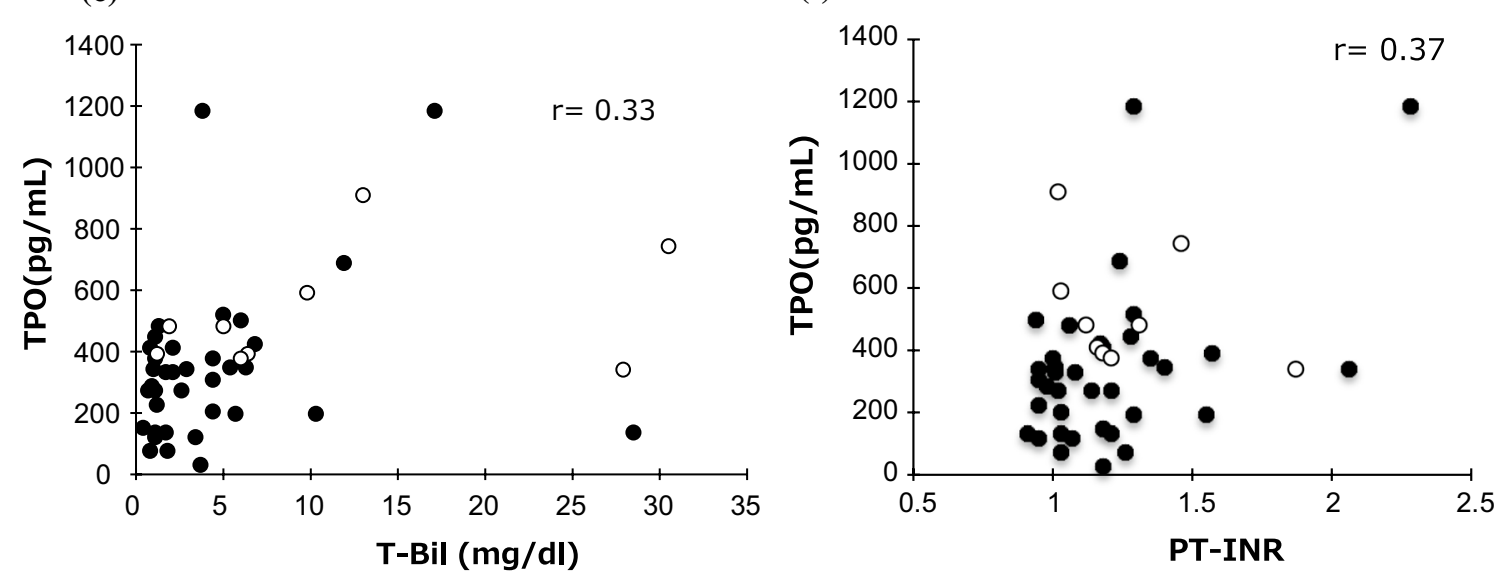

Fig. 5 Correlation between PLT and TB/PT-INR, ADAMTS13 and TB/PT-INR, and TPO levels and TB/PT-INR on POD14. The open circles designate patients who died within 6 months of LT 
Table 1 Background of the 100 adult LDLT recipients $(n=100)$

\begin{tabular}{ll}
\hline Age (year old) & $53.7(20-70)$ \\
Gender (male/female) & $61 / 39$ \\
Etiology of liver disease & \\
Liver cirrhosis (HCC) & $68(39)$ \\
PBC & 14 \\
PSC & 2 \\
Fulminant hepatitis & 11 \\
Others & 5 \\
C-P score & $9.6(5-15)$ \\
MELD score & $17.9(6-45)$ \\
Donor age (year old) & $38.0(18-65)$ \\
Graft (right/left/post) & $64 / 34 / 2$ \\
GRWR (\%) & $0.969(0.441-1.571)$ \\
WIT (min) & $48.7(21-113)$ \\
CIT (min) & $108.9(10-424)$ \\
Splenectomy & $29(29 \%)$ \\
Blood loss (ml) & $11115^{\mathrm{a}}(1426-74,480)$ \\
Transfusion & \\
RBC (unit) & $41^{\mathrm{a}}(4-213)$ \\
Platelet (unit) & $40^{\mathrm{a}}(0-120)$ \\
FFP (unit) & $40^{\mathrm{a}}(0-152)$ \\
\hline
\end{tabular}

$H C C$ hepatocellular carcinoma, $P B C$ primary biliary cirrhosis, $P S C$ primary sclerosing cholangitis, $C-P$ Child-Pugh, MELD modified end-stage liver disease, GRWR graft to recipient weight ratio, WIT warm ischemia time, $C I T$ cold ischemia time, $R B C$ red blood cells, FFP fresh frozen plasma

${ }^{\text {a }}$ Median
Table 2 Postoperative complications in LP and HP groups

\begin{tabular}{llll}
\hline & $\begin{array}{l}\text { LP group } \\
(n=36)\end{array}$ & $\begin{array}{l}\text { HP group } \\
(n=62)\end{array}$ & $P$ value \\
\hline Complication & $15(41.7 \%)$ & $12(19.4 \%)$ & $\mathbf{0 . 0 2}$ \\
Pneumonia & 5 & 3 & 0.12 \\
Sepsis & 4 & 5 & 0.33 \\
Biliary leakage & 0 & 5 & 0.10 \\
ARF & 7 & 2 & $\mathbf{0 . 0 1}$ \\
HAT & 4 & 2 & 0.13 \\
\hline
\end{tabular}

Bold values indicate statistically significant differences

$A R F$ acute renal failure (all recipients underwent renal replacement therapy), HAT hepatic artery thrombosis 
Table 3 Patient characteristics (HP and LP group)

\begin{tabular}{|c|c|c|c|c|}
\hline & All $(n=65)$ & HP group $(n=42)$ & LP group $(n=23)$ & $P$ \\
\hline \multicolumn{2}{|l|}{ Preoperative factor } & \multicolumn{3}{|l|}{ Mean \pm S.D. } \\
\hline Age (years) & $53.09 \pm 10.44$ & $52.36 \pm 11.71$ & $54.39 \pm 7.98$ & 0.461 \\
\hline Gender (male) & $39(60.0 \%)$ & $27(64.3 \%)$ & $12(52.2 \%)$ & 0.198 \\
\hline C-P score & $9.65 \pm 2.58$ & $8.69 \pm 2.32$ & $11.17 \pm 2.04$ & $<0.001$ \\
\hline MELD score & $18.30 \pm 9.57$ & $15.60 \pm 7.86$ & $22.22 \pm 10.00$ & 0.009 \\
\hline GRWR (\%) & $1.02 \pm 0.19$ & $1.04 \pm 0.19$ & $0.97 \pm 0.18$ & 0.168 \\
\hline Platelet & $75.47 \pm 61.13$ & $82.69 \pm 58.84$ & $64.17 \pm 65.34$ & 0.248 \\
\hline $\mathrm{AT}(\%)$ & $51.33 \pm 25.19$ & $58.17 \pm 25.58$ & $39.22 \pm 20.41$ & 0.003 \\
\hline ADAMTS13 (\%) & $69.09 \pm 36.96$ & $75.84 \pm 37.47$ & $59.24 \pm 32.94$ & 0.080 \\
\hline $\mathrm{vWF}(\%)$ & $285.35 \pm 156.22$ & $275.26 \pm 150.77$ & $309.30 \pm 167.97$ & 0.406 \\
\hline vWF/ADAMTS13 & $6.77 \pm 8.57$ & $5.56 \pm 5.001$ & $8.72 \pm 12.73$ & 0.158 \\
\hline \multicolumn{5}{|l|}{ Intraoperative factor } \\
\hline Splenectomy & $/(21.4 \%)$ & $9 / 42(21.4 \%)$ & $5 / 23(21.7 \%)$ & 0.606 \\
\hline CIT (min) & $110.34 \pm 73.82$ & $106.56 \pm 54.88$ & $120.17 \pm 100.09$ & 0.551 \\
\hline WIT (min) & $46.78 \pm 16.31$ & $48.76 \pm 18.09$ & $43.13 \pm 12.53$ & 0.191 \\
\hline Blood loss (ml) & $16370 \pm 15363$ & $17122 \pm 17822$ & $15379 \pm 9987$ & 0.615 \\
\hline \multicolumn{5}{|l|}{ Transfusion } \\
\hline RBC (unit) & $43.02 \pm 40.44$ & $43.26 \pm 45.42$ & $43.30 \pm 31.20$ & 0.997 \\
\hline FFP (unit) & $37.33 \pm 34.58$ & $38.33 \pm 36.94$ & $36.26 \pm 31.20$ & 0.820 \\
\hline Platelet (unit) & $33.56 \pm 27.04$ & $32.26 \pm 29.64$ & $34.78 \pm 22.08$ & 0.722 \\
\hline PVP (mmHg) & $18.92 \pm 5.05$ & $18.62 \pm 4.72$ & $19.57 \pm 5.70$ & 0.481 \\
\hline \multicolumn{5}{|c|}{ Postoperative factor (POD14) } \\
\hline TB (mg/dl) & $5.63 \pm 6.93$ & $3.38 \pm 4.92$ & $9.65 \pm 8.18$ & 0.002 \\
\hline PT-INR & $1.18 \pm 0.27$ & $1.09 \pm 0.15$ & $1.35 \pm 0.36$ & 0.003 \\
\hline CRP & $4.40 \pm 4.65$ & $3.95 \pm 4.38$ & $5.61 \pm 5.21$ & 0.192 \\
\hline $\operatorname{AT}(\%)$ & $81.49 \pm 22.11$ & $89.54 \pm 14.48$ & $67.49 \pm 26.13$ & 0.001 \\
\hline ADAMTS13 (\%) & $33.34 \pm 21.03$ & $39.94 \pm 21.22$ & $21.73 \pm 15.09$ & 0.001 \\
\hline $\operatorname{vWF}(\%)$ & $285.35 \pm 156.22$ & $343.40 \pm 100.96$ & $336.03 \pm 125.66$ & 0.802 \\
\hline vWF/ADAMTS13 & $23.31 \pm 38.47$ & $15.99 \pm 21.50$ & $35.87 \pm 55.44$ & 0.129 \\
\hline
\end{tabular}

Italic values indicate statistically significant differences

$C-P$ Child-Pugh score, MELD modified end-stage liver disease, GRWR graft to recipient weight ratio, $A T$ antithrombin, ADAMTS13 a disintegrin and metalloproteinase with a thrombospondin type 1 motifs 13 , $v W F$ von Willebrand Factor, $C I T$ cold ischemia time, WIT warm ischemia time, $R B C$ red blood cell, FFP fresh-frozen plasma, $P V P$ portal venous pressure, $T B$ total bilirubin, $P T$-INR prothrombin time international normalized ratio, $C R P C$-reactive protein 
Table 4 Risk factors for postoperative thrombocytopenia on POD14

\begin{tabular}{|c|c|c|c|c|}
\hline & \multicolumn{2}{|c|}{ Univariate analysis $(n=65)$} & \multicolumn{2}{|l|}{ Multivariate analysis } \\
\hline & Correlation coefficients & $P$ & Regression coefficient & $P$ \\
\hline \multicolumn{5}{|l|}{ Preoperative factor } \\
\hline C-P score & -0.445 & $<0.001$ & -0.088 & 0.632 \\
\hline MELD score & -0.309 & 0.012 & 0.053 & 0.296 \\
\hline Platelet & 0.362 & 0.003 & 0.032 & 0.829 \\
\hline $\mathrm{AT}(\%)$ & 0.379 & 0.003 & 0.417 & 0.002 \\
\hline \multicolumn{5}{|c|}{ Postoperative factor (POD14) } \\
\hline $\mathrm{TB}(\mathrm{mg} / \mathrm{dl})$ & -0.430 & $<0.001$ & -0.171 & 0.244 \\
\hline PT-INR & -0.324 & 0.010 & -0.122 & 0.352 \\
\hline $\mathrm{AT}(\%)$ & 0.431 & $<0.001$ & 0.212 & 0.137 \\
\hline ADAMTS13 (\%) & 0.416 & 0.001 & 0.331 & 0.011 \\
\hline
\end{tabular}

Italic values indicate statistically significant differences

$C$ - $P$ Child-Pugh score, $M E L D$ modified end-stage liver disease, $A T$ antithrombin, $T B$ total bilirubin, $P T$ $I N R$ prothrombin time international normalized ratio, ADAMTS13 a disintegrin and metalloproteinase with a thrombospondin type 1 motifs 13 